\title{
ESIN in Femur Fractures
}

\section{Exact Technique Is Important!}

\author{
Alexander Joeris', Gretel Bansi ${ }^{1}$, Peter Knorr ${ }^{2}$, Justus Lieber ${ }^{3}$, Peter Schalamon ${ }^{4}$, \\ Theddy Slongo ${ }^{1}$
}

\begin{abstract}
Background and Purpose: Elastic stable intramedullary nailing (ESIN) is gaining increasing popularity, but studies with high case loads are rare. It was the aim of four experienced pediatric trauma centers to give an update of indications for ESIN, postoperative management, and complications.

Patients and Methods: Data of the last 100 ESIN cases of each department before June 30, 2003 were collected by reviewing the charts and X-rays. Among these 400 collected ESINs 65 femoral shaft fractures $(16 \%)$ were found. The patients' age ranged between $2^{3 / 4}$ and $15^{1 / 1}$ years. The middle third of the shaft was affected 42 times (65\%), 13 fractures (20\%) were in the proximal third of the diaphysis, and ten (15\%) in the distal part of the femur, five dia- and five metaphyseal. Mainly transverse fractures were treated ( $52 \%$ ), followed by $38 \%$ oblique or spiral fractures and $10 \%$ wedge or comminuted fractures. Two open reductions were required. Median hospitalization time was 6 days. Nails were extracted after a mean of 178 days.

Results: Six skin irritations (wound infections, hematoma, seroma), one patient with myositis ossificans and one with constant pain at too long nail end were found. Three cosmetically relevant scarrings were observed during follow-up. Relevant axial deviations at fracture healing in two and shortening in one fracture could be seen, all caused by technical failure.
\end{abstract}

Conclusion: ESIN meets all demands for an optimal fracture healing in children. Still, a considerable per-

\footnotetext{
'Department of Surgical Pediatrics, Children's Hospital, University of Bern, Switzerland,

2 Department of Pediatric Surgery, Dr. von Hauner's Children's Hospital, University of Munich, Germany,

${ }^{3}$ Department of Pediatric Surgery, St. Hedwig's Hospital, Clinical Center Barmherzige Brüder, Regensburg, Germany,

4 Department of Pediatric Orthopedics, Pediatric Surgical University Hospital, Graz, Austria.
}

Received: December 21, 2004; revision accepted: January 7, 2005 centage of complications is observed, mainly caused by the surgeon himself, which can be avoided by exact indication and technique. Postoperative management has yet to be standardized.

\section{Key Words \\ ESIN · Femur fractures - Children · Complications . Indications}

Eur J Trauma 2005;31:24-32

DOI $10.1007 /$ s00068-005-1075-3

\section{Introduction}

25 years ago, the Nancy group of pediatric orthopedists introduced the new technique of elastic stable intramedullary nailing (ESIN) for stabilization of long bone fractures in children [1]. Since then it has gained great popularity. The technique was improved continuously to achieve best results. ESIN was mainly applied in diaphyseal fractures, and nowadays it can be called the gold standard for long bone diaphyseal fractures [2].

The first indication for ESIN in children was the femoral shaft fracture [3]. In the beginning, ESIN was used only for children $>10$ years of age. Today, it is widely accepted to treat children $>4$ years of age with ESIN. The various types of traction therapy, cast therapy and plating have been abandoned [3]. It is still preferred to stabilize diaphyseal fractures, but with grow- 
ing knowledge and experience the indications for ESIN are set broader and the method is used even in some metaphyseal fractures and special indications such as bone cysts $[4,5]$.

The aim of this study was to review the recent femur fractures stabilized with ESIN in four pediatric surgical departments (Bern, Switzerland; Graz, Austria; Munich, Germany; Regensburg, Germany), all having great experience with ESIN. It was our main goal to characterize the common practice in ESIN, to show the indications, and to picture the results, the still existing problems and complications as well as the long-term clinical courses. Furthermore, we try to give hints to avoid problems or complications in treating femoral fractures by ESIN.

\section{Patients and Methods}

Each of the four participating departments collected the data of their last 100 ESINs before June 30, 2003, by reviewing the charts and X-rays. All femur fractures were sent to Bern and analyzed in a standardized way.

The analysis included demographic data, place of accident, fracture type, indication for ESIN, and concomitant injuries.

Intraoperative data included operation time, intraoperative X-ray time, open or closed reduction, nail size, and additional osteosynthesis or casts.

The term "postoperative management" covered data such as length of hospital stay, duration of immobilization, time of partial and full weight bearing.

We tried to distinguish between early and long-term complications. "Early complications" implied intra- or postoperative problems like iatrogenic nerve lesions, unstable fixation or secondary loosening of nails, breakage of nails, wound infections, soft-tissue irritations or skin perforation by the nail ends, seroma or hematoma and joint effusion. "Long-term complications" described healing with axial deviation, length discrepancy, growth disturbance, persistent reduction of joint movement, malrotation, delayed union or nonunion, and cosmetic result.

\section{Results}

65 of the reviewed 400 ESINs (16.25\%) were performed for femur fractures. No bilateral fractures occurred. The male : female ratio was $46: 19$. The patients' age ranged between 2 years 9 months (minimum) and 15 years 4 months (maximum), with a mean of 8 years and 3 months. Femur fractures were mainly sustained in

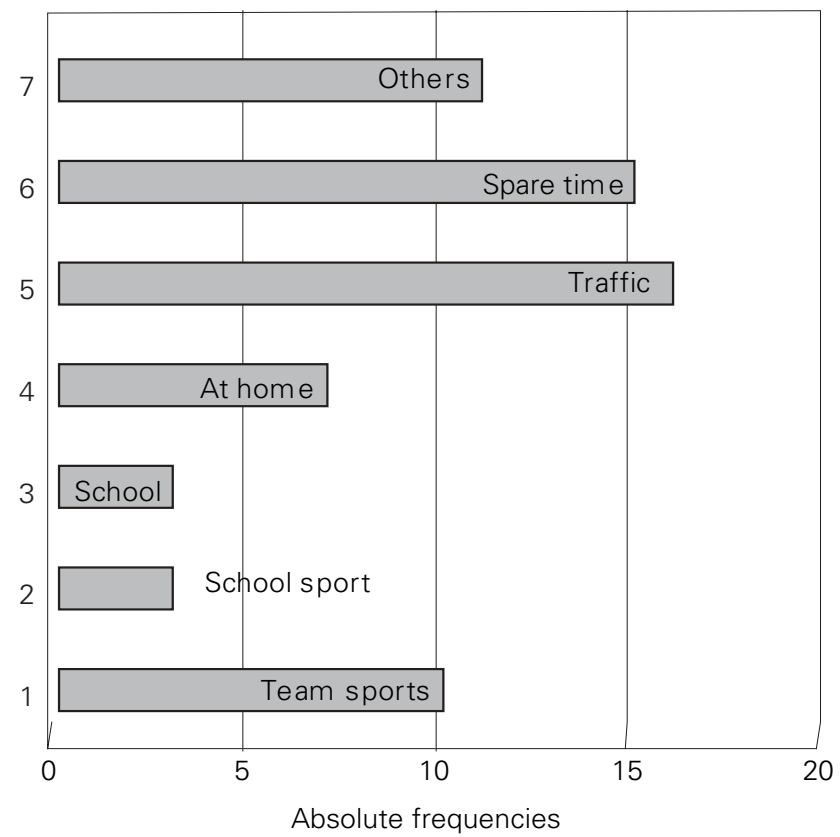

Figure 1. Scene of injury.

traffic accidents, spare-time and team sports $(25 \%$; Figure 1).

The right femur was involed 34 times (52\%), the left one 31 times $(48 \%) .43$ fractures $(67 \%)$ were located in the middle third of the diaphysis, twelve $(18 \%)$ in the proximal diaphysis, and ten $(15 \%)$ in the distal part of the femur, five diaphyseal and five metaphyseal. Five children $(7.6 \%)$ presented with a refracture: one suffered from osteogenesis imperfecta, one from enchondromatosis, one child had a transverse fracture and was treated before with a cast. Two children had femur fractures treated with plates with the refracture occurring once after plate removal and once together with a plate breakage.

64 fractures proved to be complete fractures of both cortices, including two pathologic fractures due to bone cysts. One child with a bone cyst of the proximal femur had an incompletely fractured cortex. No open fracture was observed among the reviewed cases. Table 1 shows the distribution of the different fracture types classified with the new AO-PEAG classification of long bone fractures in children.

In 62 of the cases, the indication for osteosynthesis was given by initial instability or displacement, in three patients by polytrauma. Two thirds of the fractures were stabilized on the day of injury, overall $80 \%$ were treated within $48 \mathrm{~h}$. In one multiply injured child the fracture could not be stabilized earlier than 13 days after injury. 
Table 1. Distribution of the different femur fracture types classified with the new AO classification for children's fractures.

\begin{tabular}{lrr}
\hline Classification of fractures & n & $\%$ \\
\hline Femur proximal (3 1) & & \\
- Diaphyseal transverse fracture (D4) & 5 & 7 \\
- Diaphyseal oblique/spiral fracture (D5) & 6 & 9 \\
- Diaphyseal other fractures, not classified (D5.2 and 3) & 1 & 2 \\
Femur shaft (3 2) & & \\
- Diaphyseal transverse fracture (D4) & 24 & 37 \\
Diaphyseal oblique/spiral fracture (D5) & 16 & 25 \\
- Diaphyseal other fractures, not classified (D5.2 and 3) & 3 & 5 \\
Femur distal (3 3) & & \\
- Diaphyseal transverse fracture (D4) & 2 & 3 \\
- Diaphyseal oblique/spiral fracture (D5) & 2 & 3 \\
- Diaphyseal other fractures, not classified (D5.2 and 3) & 1 & 2 \\
- Metaphyseal complete fracture (M3) & 5 & 7 \\
\hline
\end{tabular}

The standard retrograde ascending bilateral nailing was performed in 55 cases (see Figure 5), ten patients had an antegrade descending monolateral nailing because of a fracture in the distal part. Two patients needed an open reduction. In all cases two implants were used for stabilization. The diameter of the nails varied between 2.5 and $4 \mathrm{~mm}$. The nails used were either made of stainless steel $(n=39)$ or titanium $(n=26)$. Bern exclusively used titanium nails, Graz exclusively steel (Ender nails), Regensburg and Munich implanted both depending on fracture type and patient's age. In six cases high instability of the fracture and unreliable stabilization by osteosynthesis required an additional limited external fixator $(\mathrm{n}=3)$, an interlocking of the nails $(\mathrm{n}=$ $2)$, or a spica cast $(n=1)$. Table 2 gives an overview of fluoroscopy and operation time. The fluoroscopy time is divided into the four departments to show the broad range of fluoroscopy time between them.

The range of hospital stay (including the three multiply injured patients) was 2-90 days, mean 10 days. Mean start of mobilization was on day 9 (range 2-30 days). Full weight bearing was achieved after 49 days on average (range 21-150 days). Children with transverse

Table 2. Intraoperative data.

\begin{tabular}{lllc}
\hline & Mean & Minimum & Maximum \\
\hline Operation time (min) & 57 & 25 & 145 \\
Fluoroscopy time (min and s) & & & \\
- Bern & 5.01 & 1.35 & 10.35 \\
- Regensburg & 2.46 & 0.48 & 6.30 \\
Graz & 1.09 & 0.24 & 3.12 \\
Munich & 7.27 & 1.25 & 17.51 \\
\hline
\end{tabular}

and oblique/spiral fractures (AO classification D4 and D5) seemed to bear weight earlier than children with multifragmentary fractures (AO classification D5.2 and 3 ), but a statistical significance could not be found due to low case loads of fractures with three or more fragments (Figure 2).

60 patients $(92 \%)$ had a postoperative X-ray within the first 5 days after surgery. One to seven X-ray controls where performed until nail extraction (mean three $\mathrm{X}$-rays). The patients had one to seven visits (mean three visits) in the outpatient clinic before nail extraction. Bern, Munich and Regensburg saw their patients first after 4-5 weeks for radiologic proof of consolidation, Graz after 3-7 days for wound control. The nails were removed 33-372 days after implantation (mean 178 days). Differences were found between the four departments as shown in Figure 3.

Early complications occurred in nine patients $(13.8 \%)$, mainly wound infections. Two implant dislocations and one instability were documented due to incorrect placement of the nails with an insufficiently symmetrical bracing and/or due to a too small nail diameter, which can cause long-term complications such as fracture shortening or definite axial deviation (Table 3 ).

Follow-up of patients was between 7 and 35 months. Eight children (12.3\%) showed long-term complications (Table 3). Hypertrophic scars were noticed three times, once myositis ossificans was seen in a child with Lowe's syndrome, and one child complained of constant knee pain at the entry point of a too long lateral nail, so that operative cutting was necessary. Two children with

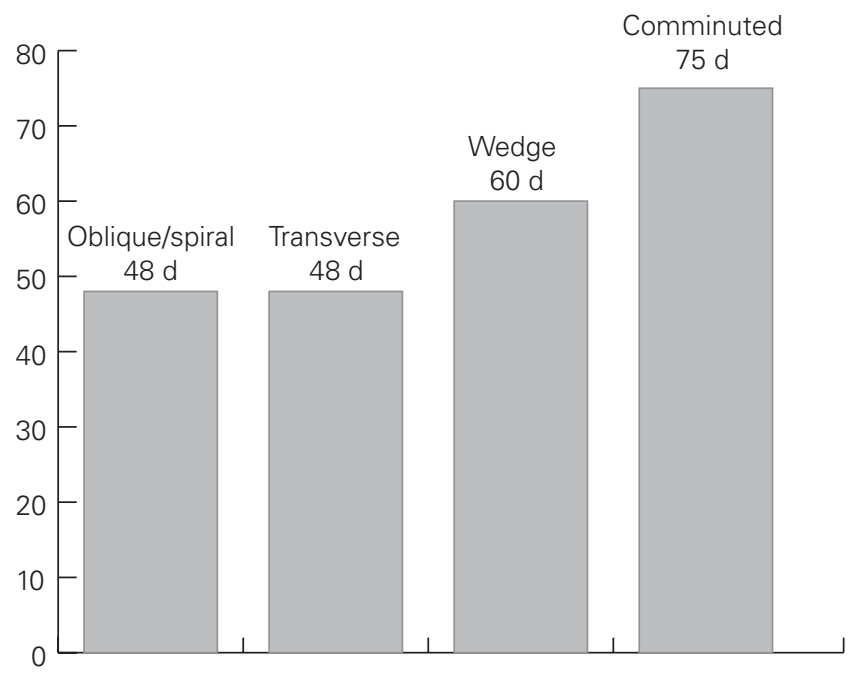

Figure 2. Days until full weight bearing on the fractured leg dependent on the type of fracture. 


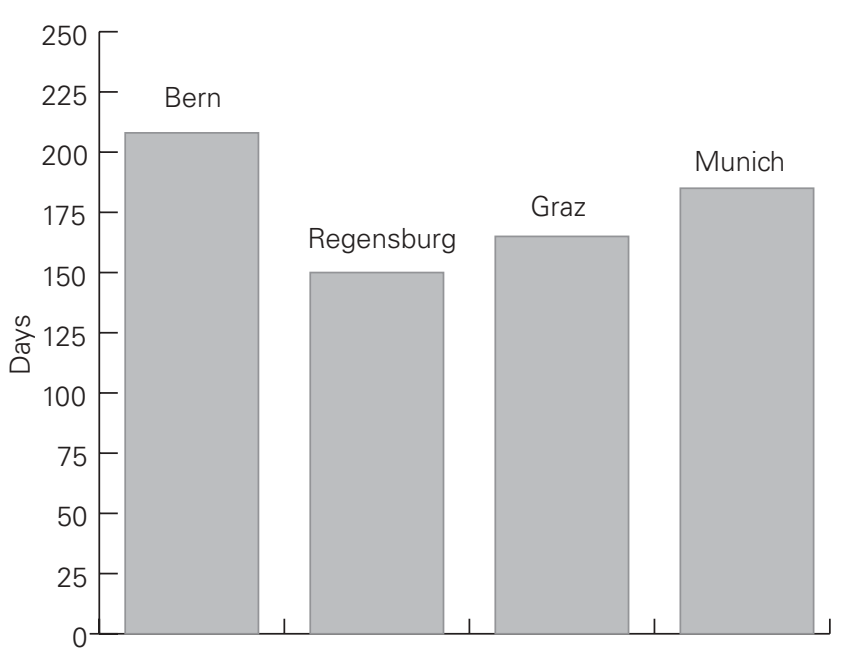

Figure 3. Period (days) until nail removal listed for each department.

Table 3. Early postoperative and long-term complications.

\begin{tabular}{lll}
\hline & $\mathbf{n}$ & $\mathbf{\%}$ \\
\hline Early postoperative complications & & \\
- Skin irritations (wound infections, hematoma, etc.) & 6 & 9.2 \\
- Implant dislocation & 2 & 3.0 \\
- Instability & 1 & 1.5 \\
Long-term complications & & \\
- Scarring (hypertrophy, distension) & 3 & 4.6 \\
- Relevant axial deviation & 2 & 3.0 \\
- Length shortening & 1 & 1.5 \\
- Myositis ossificans & 1 & 1.5 \\
- Constant pain at nail end & 1 & 1.5 \\
\end{tabular}

relevant axial deviations (defined as deviation $>10^{\circ}$ ) and one child with leg length discrepancy following postoperative shortening of the operated leg by $1 \mathrm{~cm}$ were the same as discussed before because of postoperative nail loosening and instability (Figure 4).

No iatrogenic nerve lesions, osteomyelitis, pseudarthrosis, refracture, or joint effusion were observed.

\section{Discussion}

Quite a few studies of indications, results, and complications in femur fractures treated by ESIN can be found in the literature. Most of these studies have a low case load [6-12]. There are only three studies showing a higher case load - Lascombes et al. [13] with 162 cases, Ligier et al. [3] with 123 patients, and Heinrich et al. [14] with 78 femoral shaft fractures. In 2000 a retrospective analysis of 405 femoral fractures was published by four pediatric surgery departments [2]. It represented the study with the highest case load so far. It was the aim of these four pediatric trauma centers to reevaluate their last
Figure 4. Postoperative X-ray already showing an insufficient reduction of the fracture. Length discrepancy not compensated yet. In these fractures an additional external fixator or interlocking nailing should have been the better choice.
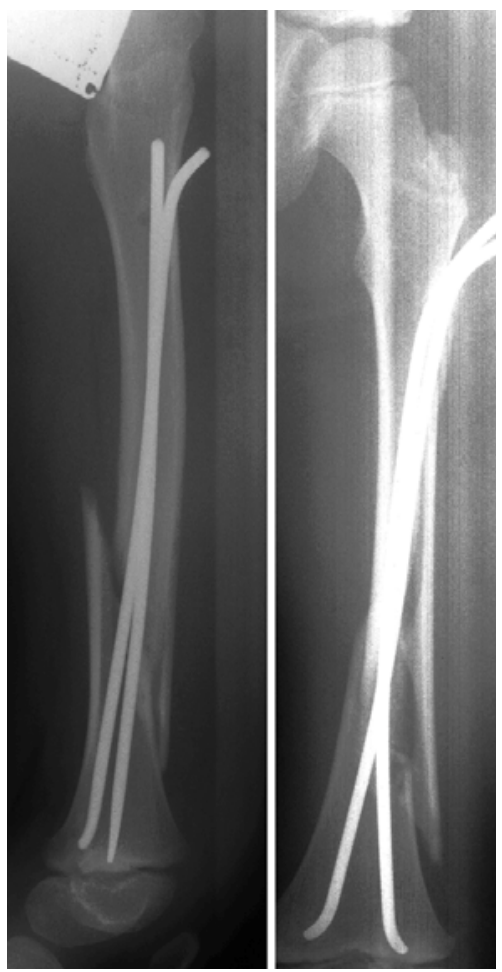

femoral fractures treated by ESIN to give an update on indications, results, early postoperative complications, and further clinical course.

Indications for ESIN in femur fractures changed within recent years concerning age and type of fracture. Reviewing the first publications about ESIN it was recommended not to use ESIN in children $<5$ years of age $[3,6]$. Other authors even recommended ESIN only for adolescents $[10,12,15]$. Main reason for this was the probable overgrowth of the fractured leg [10]. At the 19th Meeting of the Section of Pediatric Traumatology, German Society of Traumatology, in 2000 [16], the recommended age limit was children $<4$ years. Analyzing our data, we found that infants down to and seldom $<3$ years of age were treated with ESIN without showing clinically relevant overgrowth up to now. However, this is not a definitive conclusion because of the shortness of the observation period between 7 and 35 months.

In the beginning, the common indications for ESIN were transverse fractures (Figure 5), multiply injured patients and especially children with brain injuries $[6,8$, $10,17]$. Nowadays, not only all transverse femoral fractures are stabilized by intramedullary nailing (in our study $52 \%$ ) but also a majority of oblique, spiral and even some comminuted fractures. In our patient group 


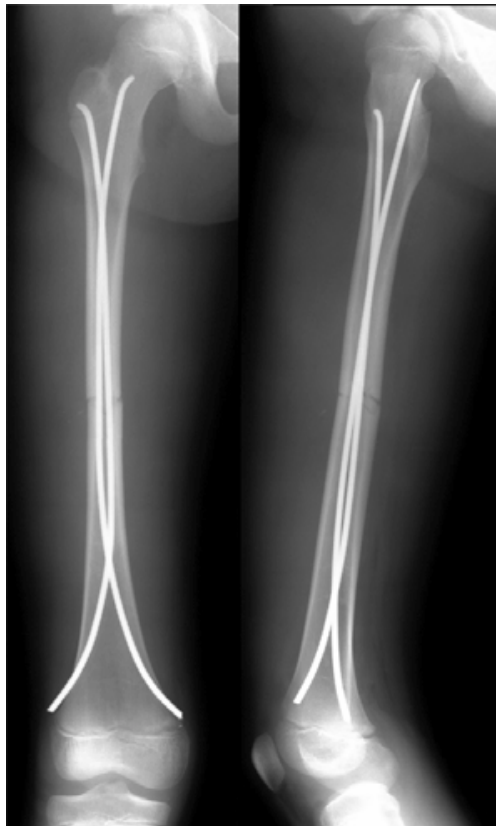

Figure 5. Typical transverse femoral midshaft fracture, one of the first indications for ESIN in children.

$48 \%$ of the fractures were oblique, spiral or comminuted fractures. For these types the most recommended treatment is external fixation [18], with the disadvantage of pin tract infections as a common complication [19-21]. It is common opinion that these kinds of fractures are insufficiently stabilized by intramedullary nailing [22-24]. In the hands of an experienced ESIN user together with the correct technique these fractures can be excellently stabilized by ESIN [25].
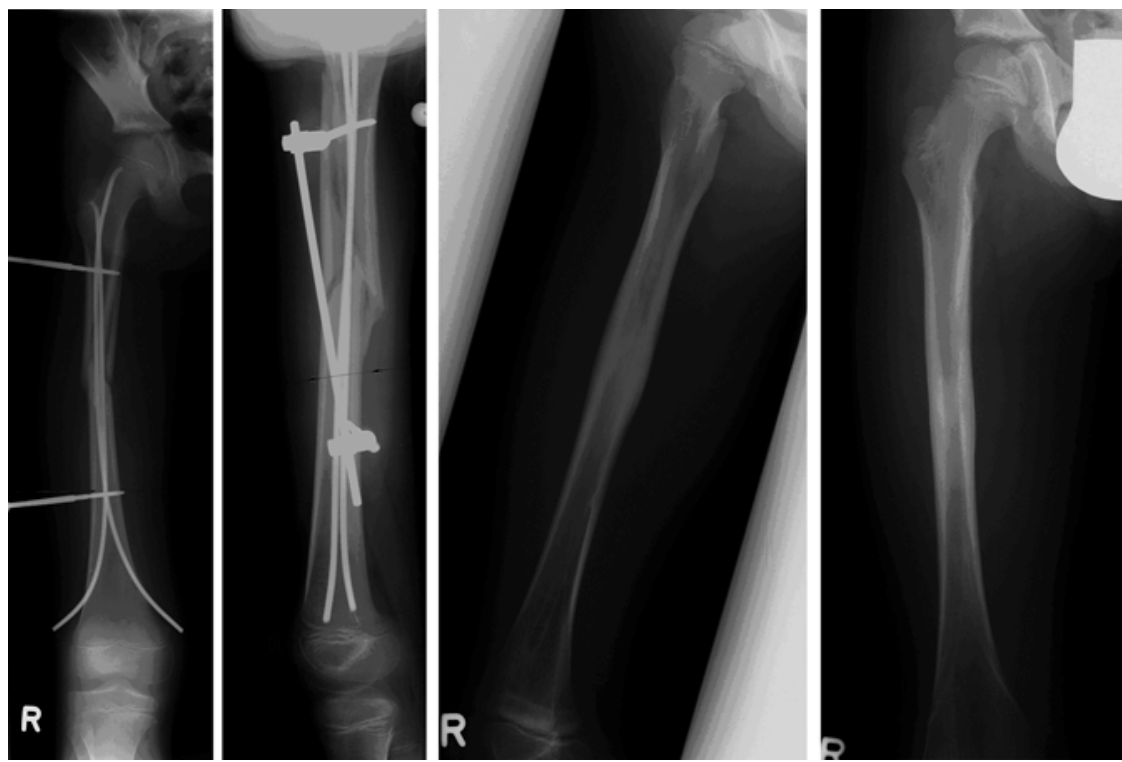

Figure 6. Combination of ESIN and external fixator. Indications are unstable oblique and comminuted fractures, which have the tendency to shortening.
One major problem and possible complication after stabilization of spiral, oblique or comminuted fractures is loss of axial stability and consecutive shortening of the fracture, if the two main fragments do not have sufficient contact and/or if a sufficient symmetrical bracing of the nails cannot be achieved. In our cases only one shortening (long oblique fracture of the distal third) and two axial deviations were observed. To avoid this, we recommend to prove the axial stability by axial blows on the distal fragment at the end of surgery. If a shortening is observed, one should verify the correct nail placement or alternatively use an additional limited external fixator with one proximal and one distal pin placed directly above and below the crossing of the nails (Figure 6). This external fixator can be removed early when first callus bridging is verified (3-4 weeks postoperatively). Interlocking of ESIN as described by Linhart \& Roposch [26] is possible in pediatric Ender nails with the interlocking holes only. In our patient group three fractures had to be stabilized with an additonal external fixator and two with interlocking. We recommend this for additional fixation rather than an additional immobilization [27].

Additionally, we found one child with a malrotation of $>10^{\circ}$, one child with an angulation of $>10^{\circ}$, and one instability. The two axial deviations have not needed further treatment so far, and the unstable reduction was additionally stabilized by a cast. Therefore, in our study $80 \%$ of all unstable fractures were treated sufficiently by ESIN alone (Figure 7). Compared with the data published by Schmittenbecher et al. in 2000 [2], the percentage of definite axial deviations could be slightly reduced, and further efforts have to be undertaken to reduce it to $<3 \%$. In order to prevent an axial deviation, the selection of the right nail diameter, the exact nail placement to achieve a threepoint support of the inner bone by each nail, but first of all the correct indication for ESIN are mandatory.

The majority of nailed femoral fractures are diaphyseal [2], but also special metaphyseal fractures can be satisfactorily stabilized by ESIN. In our collective $7 \%$ were distal metaphyseal fractures. All showed a normal consolidation without any 
axial deviation. It is mandatory that this kind of distal fractures as well as fractures in the distal diaphyseal part of the femur are stabilized by an anterograde technique. By using these techniques, an avascular necrosis of the femoral head as recently described in the literature with other nailing techniques [28-31] is avoided, because the entry of the nails is lateral subtrochanteric.

Another rare indication for ESIN are pathologic fractures in juvenile or aneurysmatic bone cyst, enchondromatosis, histiocytosis, etc. Already in 1996, Knorr et al. described the advantages of stabilizing these kinds of fractures with ESIN [4]. Among the 65 fractures analyzed, two children with pathologic fractures due to juvenile bone cysts and one child with an enchondroma of the distal femur were treated with ESIN.

One of the most significant advantages of ESIN is closed reduction, which preserves fracture hematoma, takes care of periosteum and

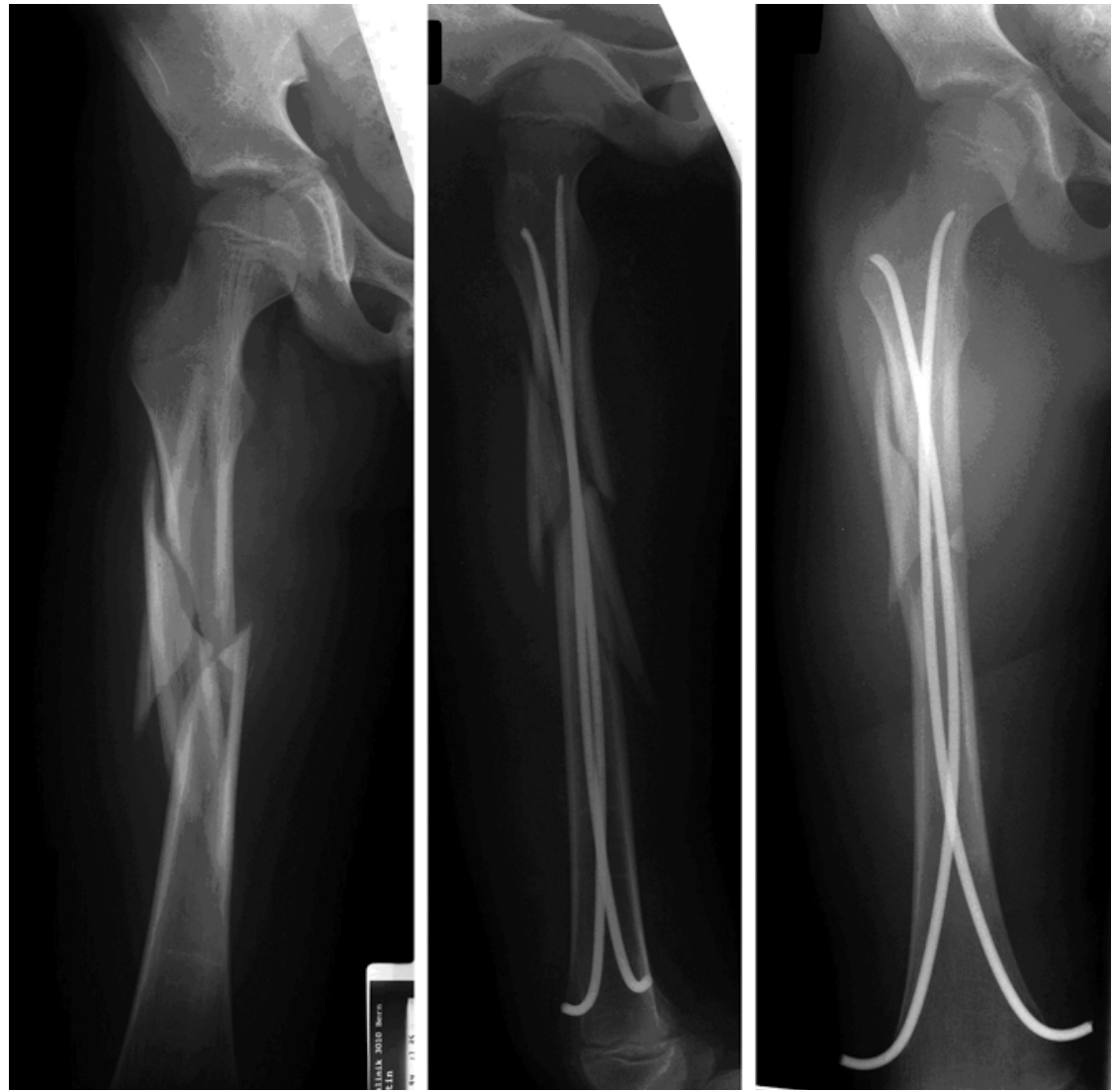

Figure 7. Unstable, long oblique spiral fracture with a third fragment which could be reduced and stabilized by ESIN alone. soft tissue in the fracture region and leads to fast periosteal healing [2]. According to earlier reports closed reduction was impossible in $4.4 \%$ up to $37 \%$, including all fracture localizations $[32,33]$. We could minimize open reduction down to $3 \%$. It is important to approximate the fracture surfaces before draping the leg. In addition, traction table, a special external reduction tool (F-Tool) or intramedullary manipulation of the distal fragment by the implanted nails are helpful.

We have to critically discuss the fluoroscopy time during surgery, which is described to be quite high, partly $>5 \mathrm{~min}$ [34]. Bar-On et al. reported an average of 2.6 min in ten femoral fractures stabilized by ESIN [8], Maier et al. had a mean intraoperative fluoroscopy time of $4.1 \mathrm{~min}$ [35]. Our mean fluoroscopy time was $4.27 \mathrm{~min}$ (0.24-17.51 $\mathrm{min}$ ) and differed between the centers (1.09 min in Graz and 7.27 min in Munich; Table 2). Despite the aspect that all four departments are teaching hospitals, this rather long fluoroscopy time reminds everybody to use fluoroscopy economically. Sometimes, an incision on the level of fracture for open reduction should rather be used earlier to minimize long fluoroscopy times. Nevertheless, it is mandatory to control the position of the nail tips in the anteroposterior and lateral view to avoid a false position of the nails. Especially a perforation of the greater trochanter by the lateral nail or the femoral neck by the medial nail should be prevented. No perforation was observed in our collective, and therefore this kind of complication could be reduced compared to 2000 [2].

There is still no agreement and the discussion is going on, which kind of material to use for ESIN. While in Bern exclusively titanium elastic nails (TEN) and in Graz exclusively modified stainless steel pediatric Ender nails are used, Regensburg and Munich use both materials. The advantage of the modified stainless steel nail used in Graz is the possibility of interlocking. Titanium nails are considered to be more flexible. Finally, the choice of nails is probably more dependent on the surgeon's preferences and experiences.

Although Hedequist et al. could not find a higher incidence of pulmonary complications in children with a 
delayed stabilization of their femoral fracture [36], a femoral fracture should be stabilized as soon as possible, because delay is often painful. Two thirds of our children underwent nailing on the day of injury, overall $80 \%$ within $48 \mathrm{~h}$.

In the literature the mean hospital stay varies between 6 and 10 days $[11,12,26]$. We found a rather long hospital stay in our group (mean 10 days [2-90 days]), which might be explained by the number of multiply traumatized children. With regard to the median hospital stay, we found a duration of 6 days.

On the 9th postoperative day on average, the children were mobilized and could walk with crutches, which is comparable to the already published data $[7$, $10,26]$. Analyzing our data in terms of duration until full weight bearing after surgery, we found differences among the four departments (33 days up to 69 days), even though no differences were found in terms of severity of fracture or concomitant injuries. These differences among the four departments might be explained by a missing agreement on the question which fracture type stands full weight bearing after how many days. For example, Regensburg allows full weight bearing of simple transverse fractures straight after surgery, whereas Bern permits only partial weight bearing first. Contrary to oblique fractures there is no contraindication to full weight bearing in simple transverse fractures, because there is no risk of secondary shortening or angulation. A full weight bearing straight after surgery might be recommended, but one has to keep in mind not to force our little patients too much because of their fear. Therefore, full weight bearing might not be achieved within the first days after the operation. Similar to recent published results the nails were explanted after a mean of 6 months (33-372 days) [8,14]. A small difference in days until nail extraction was found among the four departments, but it is difficult to give a reason for that. Once the indication for nail extraction is set, it takes up to 8 weeks in some departments because of a waiting list until the nails can be extracted in a day-surgery stay at hospital. In an uncomplicated healing process we recommend to extract the nails after 6 months.

Complications of ESIN can be divided into immediate intra- or postoperative complications or problems and long-term complications. Immediate intraoperative complications so far include iatrogenic nerve lesions, fixation in axial deviation or instability, iatrogenic bursting of an additional fragment, and nail perforation of metaphyseal bone. Immediate postoperative complica- tions are secondary loosening of nails, shortening of bone, or osteomyelitis. Postoperative local minor problems include tissue irritation, skin perforation by nail ends, major problems are wound infections, joint effusion, etc. $[9,16,37]$. Healing in deviation, length discrepancies after healing, growth disturbances, and persistent reduction of joint movement are called long-term complications.

According to the literature, early postoperative skin problems after ESIN of long bone fractures in children, such as swelling, pain, hematoma, seroma, perforation and infection, occur in $6.4-11.8 \%$ [2, 3, 32, 38]. We observed $9.2 \%$ of skin problems, mainly wound infections, but only three out of these six cases resulted in long-term problems by cosmetically unsatisfactory scars. We have to admit, that in contrast to our data from 2000 , the percentage of skin problems increased from $6.4 \%$ up to $9.2 \%$. It is claimed in the literature that $90 \%$ of the skin problems are caused by sharp nail ends [2]. Better cutting instruments and nails with a definite length and spherical ends can avoid these problems. Also, limited postoperative flexion and/or extension can be due to an incorrect shortening $[2,3,29,38]$. To avoid these problems, a sufficient incision of the fascia lata and a shortening of the lateral nail below the iliotibial tract are necessary. We found two children with limited flexion and one with limited extension after nailing; in two of the three children full movement was achieved after nail removal. To avoid irritation or swelling of the knee, the correct entry point of the nails is $2 \mathrm{~cm}$ proximal to the growth plate. This entry point guarantees not to damage the growth plate and not to irritate the capsule of the knee joint.

In 1988, Ligier et al. published data of 62 children with femoral fractures with a mean follow-up of 22 months, in which no residual angulation $>10^{\circ}$ was observed [3]. In two children we found a radiologically visible axial deviation $>10^{\circ}$, but no further treatment was required so far.

Despite the fact of a still rather high percentage of complications in ESIN, our data show that most complications are minor ones (skin irritations, scarrings) and major problems such as iatrogenic nerve lesions, osteomyelitis, pseudarthrosis, refracture, and corrective surgery could be avoided by using an exact technique.

\section{Conclusion}

By using ESIN for stabilization of femoral fractures in children, a technique is provided, which meets all de- 
mands for an optimal fracture healing in children [39]. Due to its short learning curve major complications and long-term problems such as osteomyelitis, instability, axial deviation, or length discrepancies are rare. Still, a considerable percentage of intra- and postoperative problems and complications is observed, even in specialized pediatric trauma centers. After 20 years of experience we are confident that all problems with ESIN are due to a misunderstanding/misinterpretation and/or to an incorrect planning of the operation or technical faults. Therefore, application of the exact indication and technique is essential and mandatory to avoid all of these problems. This starts with an exact indication for ESIN dependent on the patient's age as well as the type and site of the fracture. Using a combination of ESIN and external fixation or interlocking nailing provides an excellent technique for stabilization, if instability, malrotation, angulation or length shortening are suspected. Small incision and "minimal open reduction" should be considered, if closed reduction seems impossible, and thereby intraoperative fluoroscopy time could be minimized. Postoperative management, such as duration until full weight bearing, number of controls in the outpatient clinic and number of X-ray controls until nail removal, have yet to be standardized.

\section{References}

1. Prevot J, Lascombes P, Ligier JN. The ECMES Centro-Medullary Elastic Stabilising Wiring osteosynthesis method in limb fractures in children. Principle, application on the femur. Apropos of 250 fractures followed-up since 1979. Chirurgie 1993;119:473-6.

2. Schmittenbecher PP, Dietz HG, Linhart WE, et al. Complications and problems in intramedullary nailing of children's fractures. Eur J Trauma 2000;26:287-93.

3. Ligier JN, Metaizeau JP, Prevot J, et al. Elastic stable intramedullary nailing of femoral shaft fractures in children. J Bone Joint Surg Br 1988;70:74-7.

4. Knorr P, Schmittenbecher PP, Dietz HG. Treatment of pathological fractures of long tubular bones in childhood using elastic stable intramedullary nailing. Unfallchirurg 1996;99:410-4.

5. Knorr P, Schmittenbecher PP, Dietz HG. Elastic stable intramedullary nailing for the treatment of complicated juvenile bone cysts of the humerus. Eur J Pediatr Surg 2003;13:44-9.

6. Rehli V, Slongo T. Prevot's method of elastic-stable endomedullary fixation - an ideal method for the management of juvenile shaft fractures. Z Unfallchir Versicherungsmed 1991;84:177-81.

7. Huber RI, Keller HW, Huber PM, et al. Flexible intramedullary nailing as fracture treatment in children. J Pediatr Orthop 1996;16:602-5.

8. Bar-On E, Sagiv S, Porat S. External fixation or flexible intramedullary nailing for femoral shaft fractures in children. A prospective, randomised study. J Bone Joint Surg Br 1997;79:975-8.

9. Till H, Huttl B, Knorr P, et al. Elastic stable intramedullary nailing (ESIN) provides good long-term results in pediatric long-bone fractures. Eur J Pediatr Surg 2000;10:319-22.
10. Mann DC, Weddington J, Davenport K. Closed Ender nailing of femoral shaft fractures in adolescents. J Pediatr Orthop 1986;6:651-5.

11. Prokop A, Jubel A, Hahn U, et al. Intramedullary fixation of pediatric bone shaft fractures. Kongressbd Dtsch Ges Chir Kongr 2002;119:526-31.

12. Gregory P, Sullivan JA, Herndon WA. Adolescent femoral shaft fractures: rigid versus flexible nails. Orthopedics 1995;18:645-9.

13. Lascombes P, Prevot J, Poncelet T, et al. Complications of stable flexible wiring in the treatment of femoral fractures in children. Rev Chir Orthop Reparatrice Appar Mot 1988;74:Suppl 2:293-6.

14. Heinrich SD, Drvaric DM, Darr K, et al. The operative stabilization of pediatric diaphyseal femur fractures with flexible intramedullary nails: a prospective analysis. J Pediatr Orthop 1994;14:501-7.

15. Greene WB. Displaced fractures of the femoral shaft in children. Unique features and therapeutic options. Clin Orthop 1998;353:86-96.

16. Dietz HG, Joppich I, Marzi I, et al. Treatment of femoral fractures in childhood. Consensus Report of the 19th Meeting of the Child Traumatology Section of the DGU, Munich, 23-24 June 2000. Unfallchirurg 2001;104:788-90.

17. Ziv I, Rang M. Treatment of femoral fracture in the child with head injury. J Bone Joint Surg Br 1983;65:276-8.

18. De Sanctis N, Gambardella A, Pempinello C, et al. The use of external fixators in femur fractures in children.J Pediatr Orthop 1996;16:613-20.

19. Gregory P, Pevny T, Teague D. Early complications with external fixation of pediatric femoral shaft fractures. J Orthop Trauma 1996;10:191-8.

20. Blasier RD, Aronson J, Tursky EA. External fixation of pediatric femur fractures. J Pediatr Orthop 1997;17:342-6.

21. Davis TJ, Topping RE, Blanco JS. External fixation of pediatric femoral fractures. Clin Orthop 1995;318:191-8.

22. Carey TP, Galpin RD. Flexible intramedullary nail fixation of pediatric femoral fractures. Clin Orthop 1996;332:110-8.

23. Winquist RA, Hansen ST Jr. Comminuted fractures of the femora shaft treated by intramedullary nailing. Orthop Clin North Am 1980;11:633-48.

24. Winquist RA, Hansen ST Jr., Clawson DK. Closed intramedullary nailing of femoral fractures. A report of five hundred and twenty cases. J Bone Joint Surg Am 1984;66:529-39.

25. Dietz HG, Schmittenbecher PP, Illing P. Intramedulläre Osteosynthese im Wachstumsalter. München: Urban \& Schwarzenberg, 1997.

26. Linhart WE, Roposch A. Elastic stable intramedullary nailing for unstable femoral fractures in children: preliminary results of a new method. J Trauma 1999;47:372-8.

27. Narayanan UG, Hyman JE, Wainwright AM, et al. Complications of elastic stable intramedullary nail fixation of pediatric femoral fractures, and how to avoid them.J Pediatr Orthop 2004;24:363-9.

28. O'Malley DE, Mazur JM, Cummings RJ. Femoral head avascular necrosis associated with intramedullary nailing in an adolescent. J Pediatr Orthop 1995;15:21-3.

29. Parsch KD. Modern trends in internal fixation of femoral shaft fractures in children. A critical review. J Pediatr Orthop B 1997;6:117-25.

30. Beaty JH, Austin SM, Warner WC, et al. Interlocking intramedullary nailing of femoral-shaft fractures in adolescents: preliminary results and complications. J Pediatr Orthop 1994;14:178-83.

31. Astion DJ, Wilber JH, Scoles PV. Avascular necrosis of the capital femoral epiphysis after intramedullary nailing for a fracture of the femoral shaft. A case report. J Bone Joint Surg Am 1995;77:1092-4.

32. Lascombes P, Prevot J, Ligier JN, et al. Elastic stable intramedullary nailing in forearm shaft fractures in children: 85 cases. J Pediatr Orthop 1990;10:167-71. 
33. Ligier JN, Metaizeau JP, Prevot J, et al. Elastic stable intramedullary pinning of long bone shaft fractures in children. $Z$ Kinderchir 1985;40:209-12.

34. Schlickewei W, Huber-Lang M, Friedel HP. Gibt es neue Behandlungserkenntnisse bei Frakturen der unteren Extremität im Kindesalter? Trauma Berufskrankh 1999;1:80-7.

35. Maier M, Maier-Heidkamp P, Lehnert M, et al. Results of femoral shaft fractures in childhood in relation to different treatment modalities. Unfallchirurg 2003;106:48-54.

36. Hedequist D, Starr AJ, Wilson P, et al. Early versus delayed stabilization of pediatric femur fractures: analysis of 387 patients. J Orthop Trauma 1999;13:490-3.

37. Schmittenbecher PP. Complications and errors in use of intramedullary nailing in shaft fractures in childhood. Kongressbd Dtsch Ges Chir Kongr 2001;118:435-7.

38. Linhart WE, Spendel S, Mayr H, et al. Die elastisch stabile intramedulläre Schienung kindlicher Schaftfrakturen. Zentralbl Kinderchir 1992;1:215-20.

39. Laer L von. Frakturen und Luxationen im Wachstumsalter. Stuttgart-New York: Thieme, 2001.
Address for Correspondence

Alexander Joeris, MD

Department of Surgical Pediatrics

Children's Hospital

Inselspital

University of Bern

Switzerland

3010 Bern

Switzerland

Phone (+41/31) 632-9223, Fax -9292

e-mail: alexander.joeris@insel.ch 